Erratum

\title{
Erratum to "Adequacy of Semitendinosus Tendon Alone for Anterior Cruciate Ligament Reconstruction Graft and Prediction of Hamstring Graft Size by Evaluating Simple Anthropometric Parameters"
}

\author{
S. G. Papastergiou, ${ }^{1}$ G. A. Konstantinidis, ${ }^{1}$ K. Natsis, ${ }^{2}$ \\ E. Papathanasiou, ${ }^{2}$ N. Koukoulias, ${ }^{1}$ and A. G. Papadopoulos ${ }^{1}$ \\ ${ }^{1}$ Orthopaedic Department of "Saint Paul" General Hospital of Thessaloniki Greece, Ethnikis Antistaseos 161, 55134 Thessaloniki, Greece \\ ${ }^{2}$ Department of Anatomy, Medical School, Aristotle University of Thessaloniki, University Campus, P.O. Box 300, \\ 54624 Thessaloniki, Greece \\ Correspondence should be addressed to G. A. Konstantinidis; konstantigeo@yahoo.com
}

Received 24 March 2013; Accepted 24 March 2013

Copyright (c) 2013 S. G. Papastergiou et al. This is an open access article distributed under the Creative Commons Attribution License, which permits unrestricted use, distribution, and reproduction in any medium, provided the original work is properly cited.

The authors' names were incorrectly listed as Papastergiou G. Stergios, Konstantinidis A. Georgios, Natsis Konstantinos, Papathanasiou Efthymia, Koukoulias Nikolaos, and Papadopoulos G. Alexandros; this error is corrected here. 

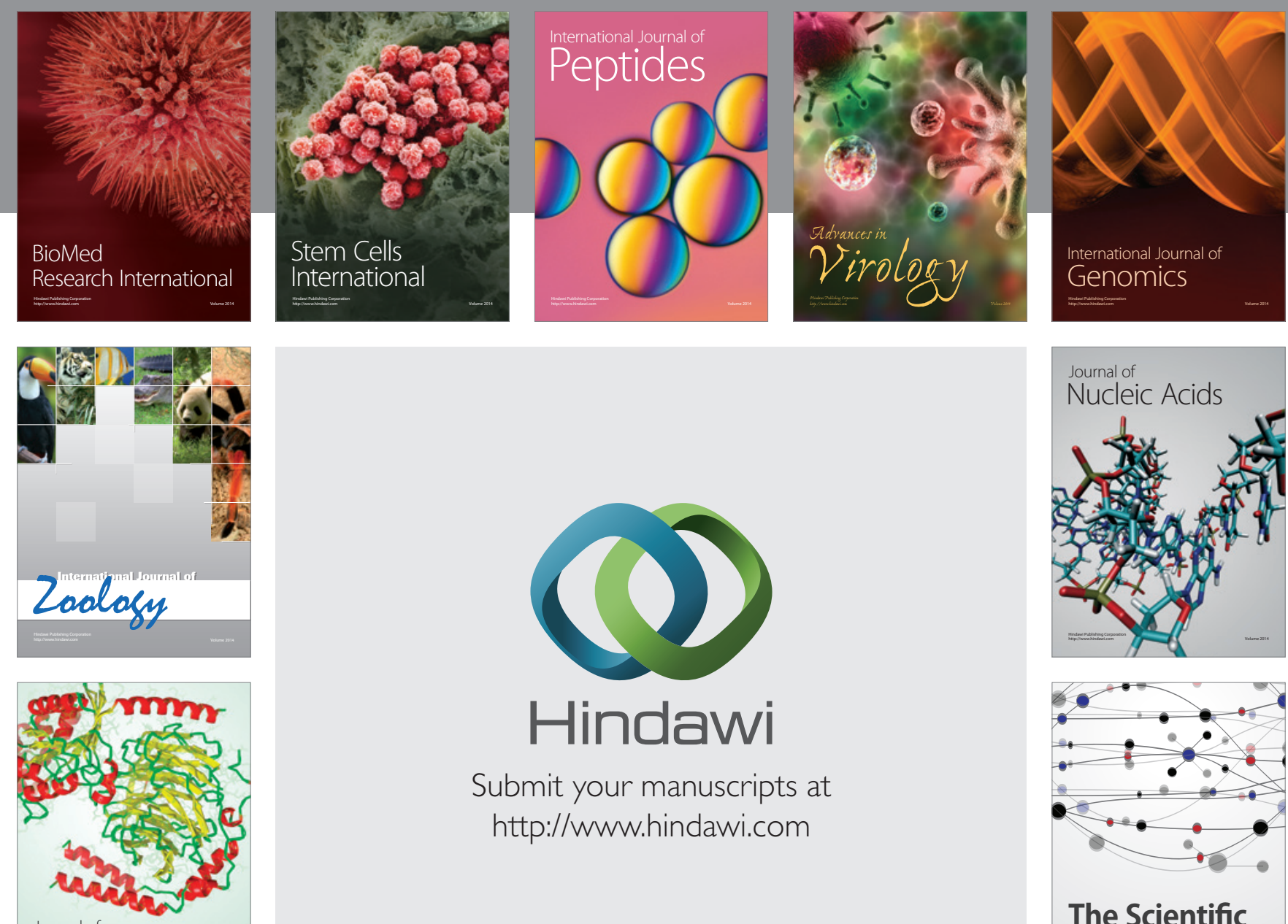

Submit your manuscripts at

http://www.hindawi.com

Journal of
Signal Transduction
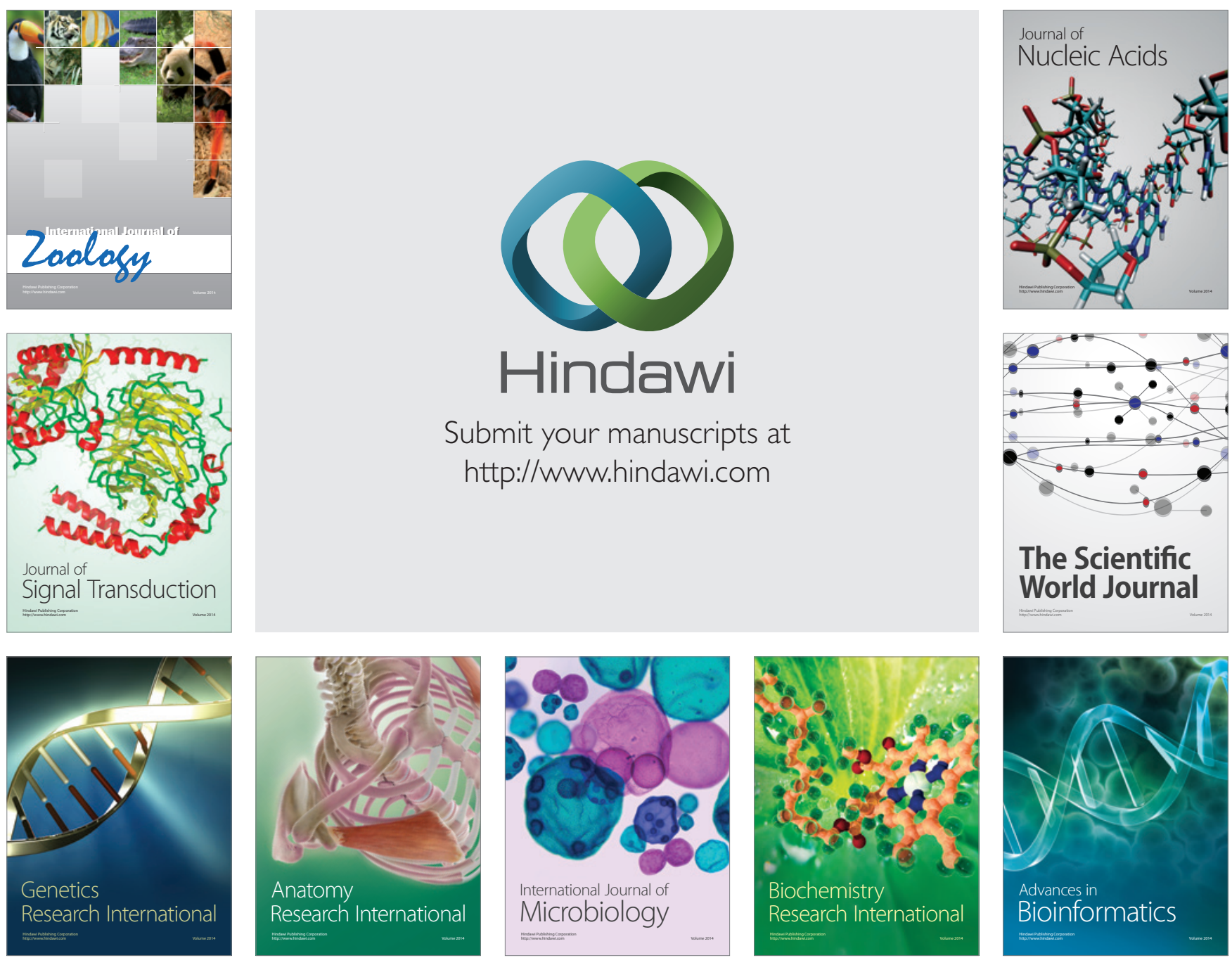

The Scientific World Journal
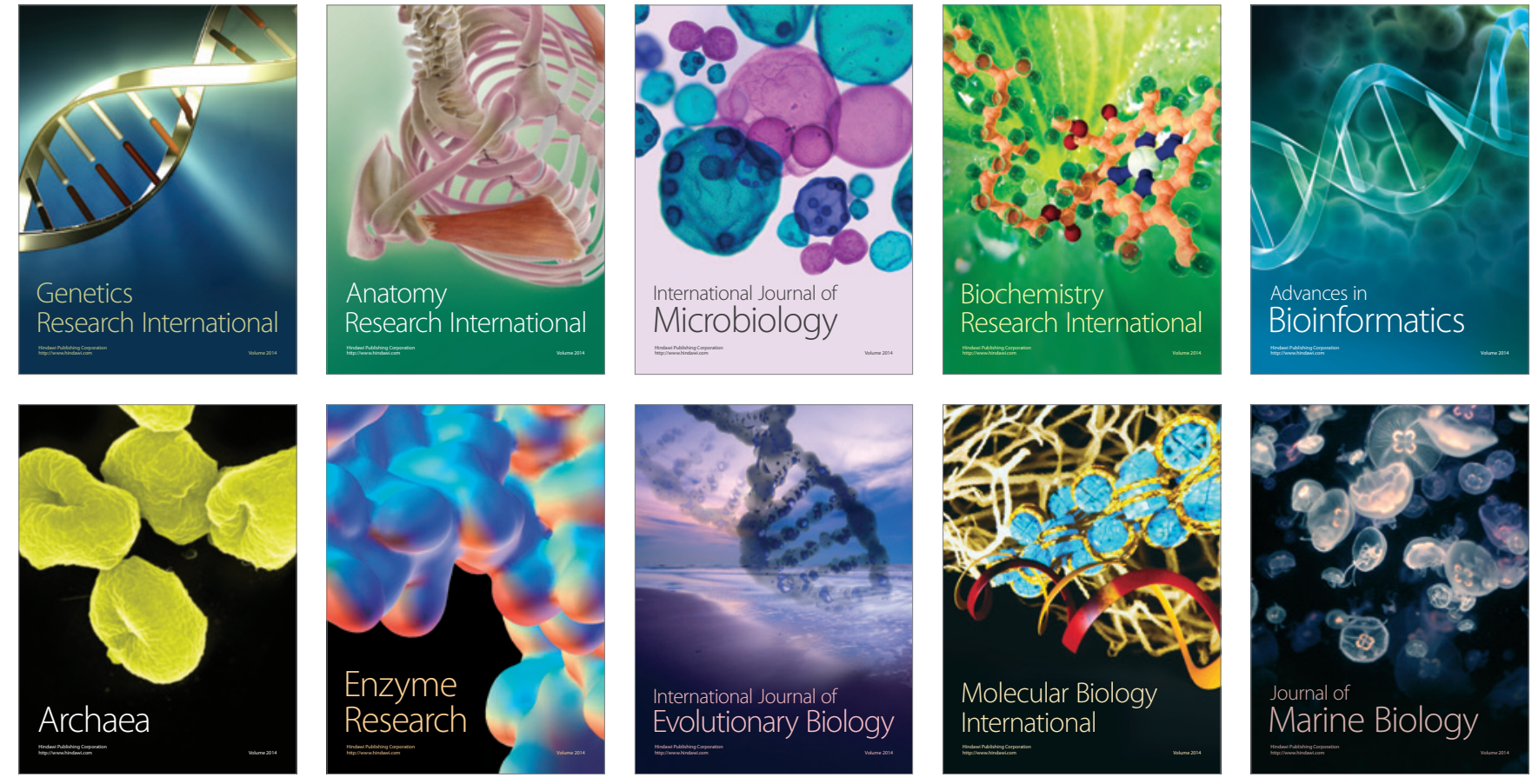